\title{
Editorial
}

\section{Advanced Composites with Natural Reinforcement}

\author{
Jim Low, ${ }^{1}$ Ian J. Davies, ${ }^{2}$ Yu Dong, ${ }^{2}$ Shaikh Faiz Uddin Ahmed, ${ }^{3}$ \\ Hao Wang, ${ }^{4}$ and Hazizan Md Akil ${ }^{5}$ \\ ${ }^{1}$ Department of Applied Physics, Curtin University, G.P.O. Box U1987, Perth, WA 6845, Australia \\ ${ }^{2}$ Department of Mechanical Engineering, Curtin University, G.P.O. Box U1987, Perth, WA 6845, Australia \\ ${ }^{3}$ Department of Civil Engineering, Curtin University, G.P.O. Box U1987, Perth, WA 6845, Australia \\ ${ }^{4}$ Centre of Excellence in Engineered Fibre Composites (CEEFC), University of Southern Queensland, Toowoomba, \\ QLD 4350, Australia \\ ${ }^{5}$ School of Materials and Mineral Resources Engineering, Universiti Sains Malaysia, 14300 Nibong Tebal, Penang, Malaysia \\ Correspondence should be addressed to Jim Low; j.low@curtin.edu.au
}

Received 12 December 2013; Accepted 12 December 2013; Published 22 April 2014

Copyright (c) 2014 Jim Low et al. This is an open access article distributed under the Creative Commons Attribution License, which permits unrestricted use, distribution, and reproduction in any medium, provided the original work is properly cited.

The strict environmental regulations and depletion of petroleum resources urge the use of alternative eco-friendly natural reinforcements to produce advanced composites. These natural fillers or fibres such as sisal, coir, basalt, hemp, flax, and wool offer great renewability, biodegradability, abundance, cost effectiveness, and low specific gravity as opposed to synthetic fibres like glass and carbon. Composite industry realises enormous advantages of their composites in better functional or structural properties over synthetic counterparts despite not having relatively high mechanical performance.

The popular research theme for composites with natural reinforcements generally covers polymer composites, geopolymer composites, ceramic composites reinforced with natural fibres and fillers (including some nano- or microfillers), fibre surface modification for the optimisation of mechanical properties, and processing of natural, renewable, and biodegradable reinforcements. This special issue aims to provide a platform for scientists, material engineers, researchers, and composite industrialists to exchange and share ideas and findings in this exciting field. There are totally six papers contained in this special issue to deal with the processing, characterisation, and properties of composites reinforced with various forms of natural fibres and fillers. The synopsis of each paper is as follows.
In "Effect of zeolite modification via cationic exchange method on mechanical, thermal, and morphological properties of ethylene vinyl acetate/zeolite composites," surface modification of zeolite by organic modifier was found to be capable of improving the properties of EVA/zeolite composites.

In "Pretreatment of woven jute FRP composite and its use in strengthening of reinforced concrete beams in flexure," woven jute fibre reinforced polymer composite was shown to be a suitable material which could be used for flexural upgradation of reinforced concrete beams.

In "Efficacy of thermally conditioned sisal FRP composite on the shear characteristics of reinforced concrete beams," woven sisal fibre reinforced polymer composite was found to impart ductile failure and increased the shear strength and initial crack load of reinforced concrete beams.

In "Nanofibre electrospinning poly (vinyl alcohol) and cellulose composite mats obtained by use of a cylindrical electrode," submicron PVA fibre mats reinforced with cellulose nanofibres were successfully prepared by the electrospinning of aqueous PVA solutions.

In "Improvement of mechanical properties of noil hemp fiber reinforced polypropylene composites by resin modification and fiber treatment," resin modification and fibre treatment have been shown to be effective in improving interfacial bonding and enhancing the mechanical properties of composites. 
In "Investigation of mechanical and structural properties of blend lignin-PMMA," lignin was found to increase the impact strength but reduce the transverse strength and elastic modulus of PMMA resin.

\section{Acknowledgments}

We would like to thank all the authors who have submitted their papers to this special issue and also to all the reviewers for their invaluable contributions to the reviewing process.

Jim Low

Ian J. Davies Yu Dong

Shaikh Faiz Uddin Ahmed Hao Wang Hazizan Md Akil 

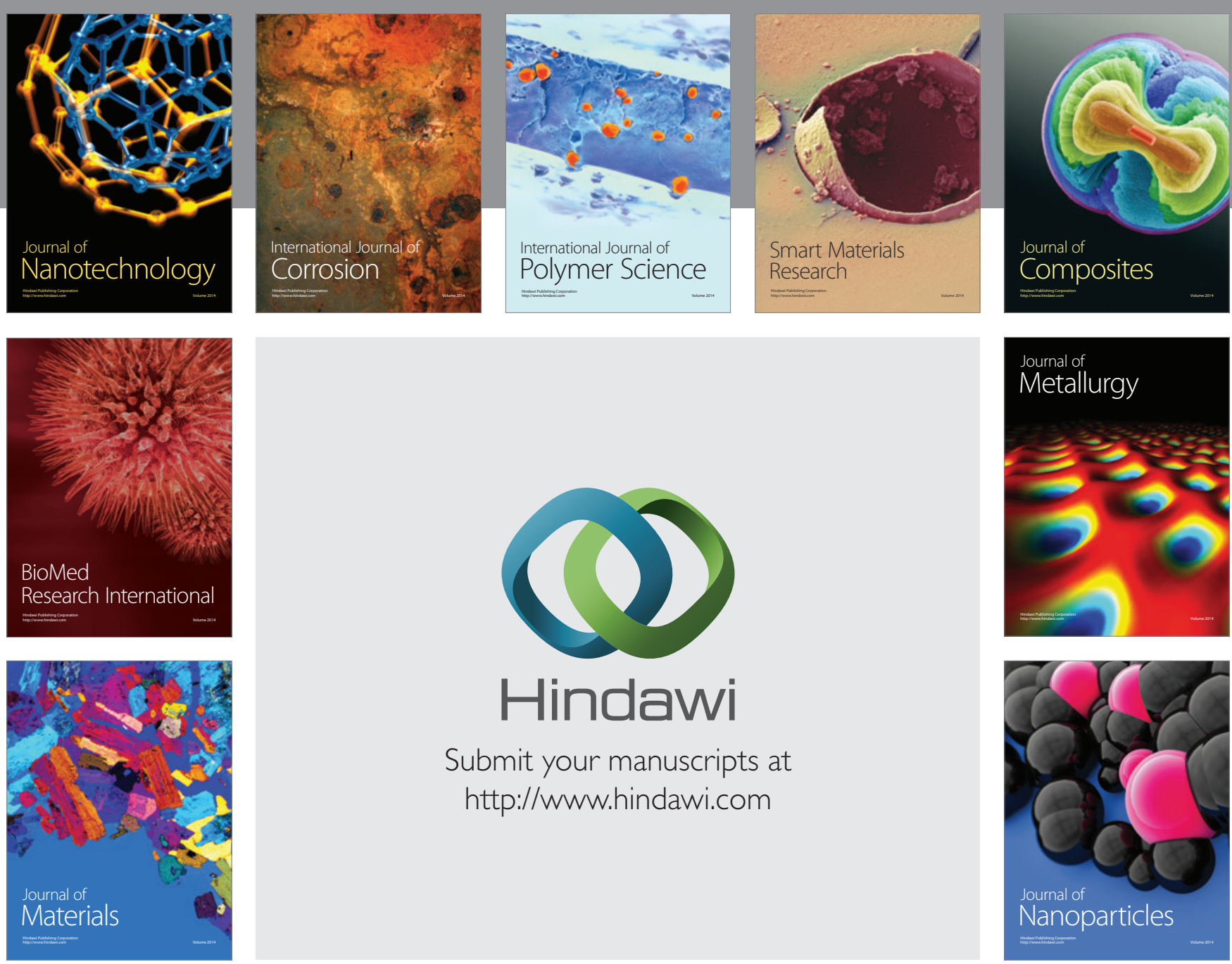

Submit your manuscripts at http://www.hindawi.com
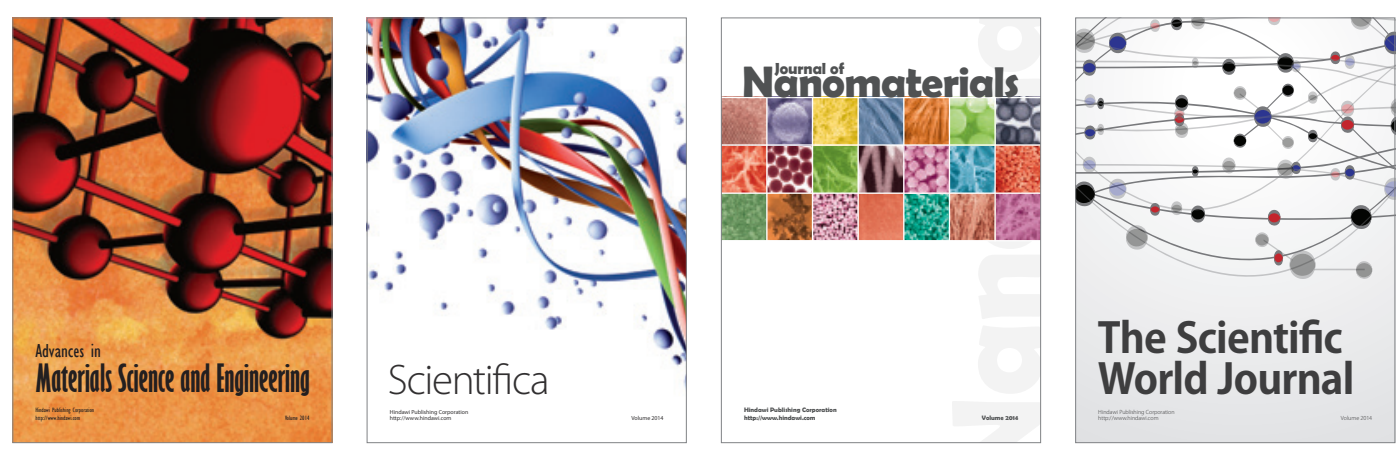

\section{The Scientific World Journal}
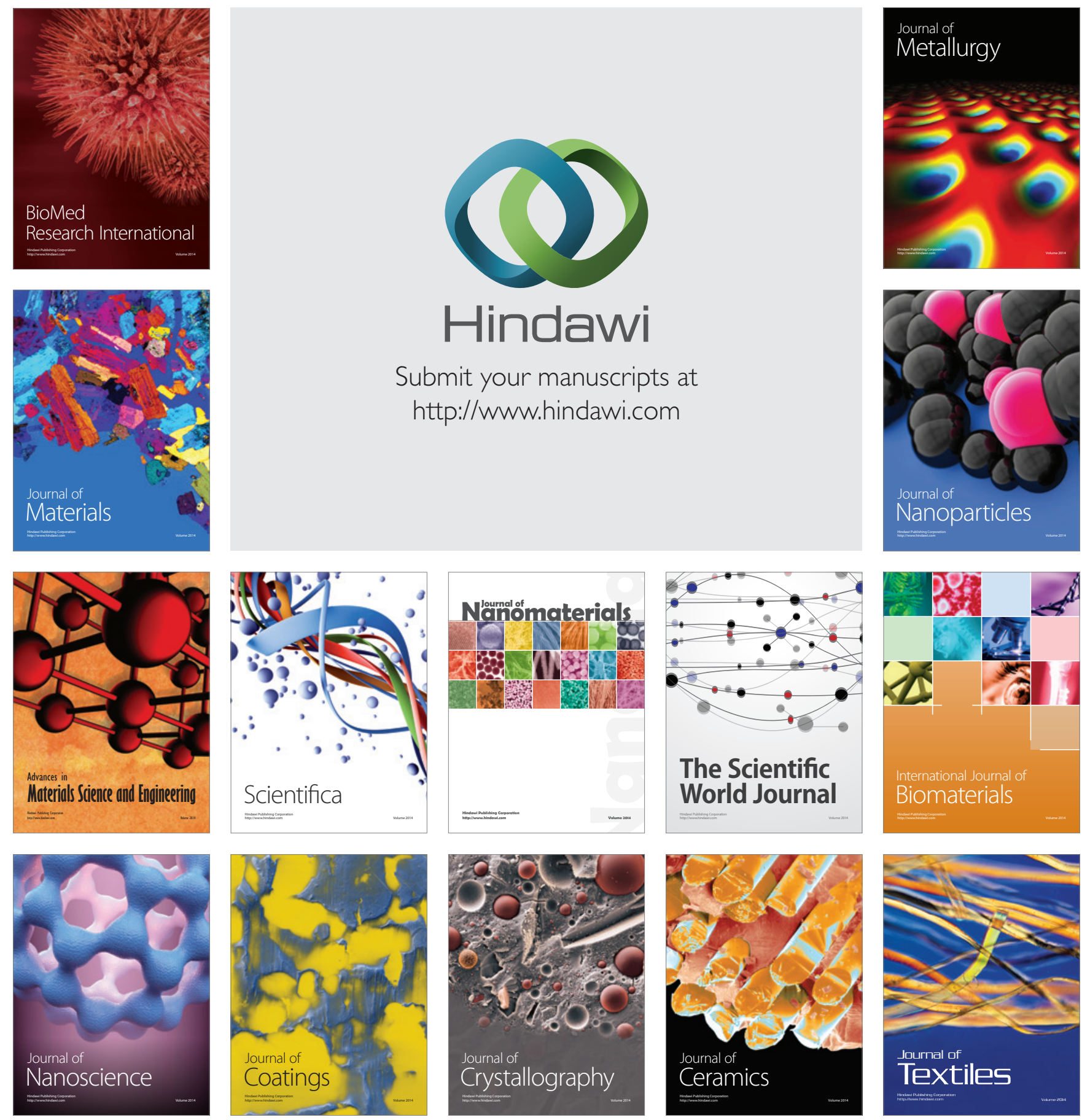